Gynecology

\title{
Discontinuation of long-acting reversible contraception versus short-term hormonal methods in urban Ghana: A pilot longitudinal study
}

\author{
Sarah D. Rominski ${ }^{1, *}$ | Abubakar Manu ${ }^{2}$ | Ernest Maya ${ }^{2}$ | Emmanuel S.K. Morhe ${ }^{3}$ | \\ Vanessa K. Dalton ${ }^{1}$
}

\author{
${ }^{1}$ Program on Women's Healthcare Effectiveness Research, Department of Obstetrics and Gynecology, University of Michigan, Ann Arbor, MI, USA \\ ${ }^{2}$ Department of Population, Family and Reproductive Health, University of Ghana School of Public Health, Legon, Ghana \\ ${ }^{3}$ Department of Obstetrics and Gynecology, University of Health and Allied Sciences, Ho, Ghana \\ ${ }^{*}$ Correspondence \\ Sarah D. Rominski, Program on Women's Healthcare Effectiveness Research, Department of Obstetrics and Gynecology, University of Michigan, Ann Arbor, \\ MI, USA. \\ Email: sarahrom@med.umich.edu \\ Funding Information \\ Institute for Research on Women and Gender at the University of Michigan
}

KEYWORDS : Contraceptive continuation; Ghana; Long-acting reversible contraception

Rates of contraceptive discontinuation are high worldwide, ${ }^{1}$ including in Ghana. ${ }^{2}$ Although long-acting reversible contraceptive (LARC) methods are widely available and safe for most women, ${ }^{3}$ few Ghanaian women use them. ${ }^{4}$ We aimed to prospectively assess whether discontinuation rates differed among women who adopted a LARC method versus those who adopted a short-term hormonal method.

Between June 1 and August 31, 2015, we recruited women aged 18 years or older who were seeking contraception at three family planning clinics in Accra, Ghana. The study was reviewed and approved by the ethical review boards of the Ghana Health Service and the University of Michigan. All women provided comprehensive verbal consent to participate.

Participants were asked about their preferences and adoption of contraceptive method both before and after family planning counseling. During follow-up phone interviews at months 3, 6, 9, and 12, participants were asked if they were still using the method they initially adopted and, in hindsight, whether they would adopt the same method again. Participants with 9- or 12-month follow-up data were combined for analysis. We used $\chi^{2}$ analysis to determine differences in continuation at 9-12 months between women who adopted a LARC method and those who adopted a short-term hormonal method.
Analysis was done with Stata version 13 (StataCorp, College Station, TX, USA). Given the low frequency of discontinuation outcomes, use of inferential statistics controlling for confounding variables was not possible.

Of 151 women who adopted a LARC or short-term method, $87(57.6 \%)$ women were retained in the study at 9-12 months (Table 1). The 9-12 month continuation rate was almost 95\% in the LARC group compared with $71.0 \%$ in the short-term hormonal group ( $P=0.002$ ). Most participants said they would choose the same method again, with no significant difference in proportions between groups $(P=0.461)$.

In this pilot longitudinal study, participants who adopted a LARC method were more likely to still be using that method at 9-12 months than were those who did not. Moreover, LARC users were just as likely to say they would choose the same method again, suggesting satisfaction. Although the retention rate was fairly low, likely due to no incentives being provided for continued participation, these preliminary data suggest that there could be important differences in continuation rates between methods. Whilst this difference could be due to LARC methods being more difficult to discontinue, it will be important to more fully investigate reasons why Ghanaian women continue or discontinue contraception. 
TABLE 1 Retention and continuation rates by method. ${ }^{a}$

\begin{tabular}{|c|c|c|c|c|c|c|}
\hline Method & Number adopted & $\begin{array}{l}\text { Retained at } \\
9-12 \text { months }\end{array}$ & $\begin{array}{l}\text { Still using at } \\
9-12 \text { months }\end{array}$ & \multicolumn{3}{|c|}{ Would use this method again } \\
\hline \multicolumn{7}{|l|}{ LARC } \\
\hline Implant & 63 & $40 / 63(63.5)$ & $38 / 40(95.0)$ & $26 / 40(65.0)$ & $14 / 40(35.0)$ & 0 \\
\hline IUD & 24 & $16 / 24(66.7)$ & $15 / 16(93.8)$ & $10 / 16(62.5)$ & $4 / 16(25.0)$ & $2 / 16(12.5)$ \\
\hline Combined & 87 & $56 / 87(64.4)$ & $53 / 56(94.6)$ & $36 / 56(64.3)$ & $18 / 56(32.1)$ & $2(3.6)$ \\
\hline Injection & 53 & $24 / 53(45.3)$ & $18 / 24(75.0)$ & $16 / 24(66.7)$ & $6 / 24(25.0)$ & $2 / 24(8.3)$ \\
\hline Pill & 11 & $7 / 11(63.6)$ & $4 / 7(57.1)$ & $6 / 7(85.7)$ & $1 / 7(14.2)$ & 0 \\
\hline Combined & 64 & $31 / 64(48.4)$ & $22 / 31(71.0)$ & $22 / 31(70.1)$ & $7(22.6)$ & $2(6.5)$ \\
\hline
\end{tabular}

Abbreviations: LARC, long-acting reversible contraceptive; IUD, intrauterine device.

${ }^{a}$ Values are given as absolute number or number/number available (percentage).

\section{AUTHOR CONTRIBUTIONS}

All authors conceived the study. AM, ESKM, and EM oversaw data collection. SDR managed and analyzed the data, and wrote the first draft of the paper. All authors reviewed and revised the manuscript.

\section{ACKNOWLEDGMENTS}

The present study was funded by the Institute for Research on Women and Gender at the University of Michigan.

\section{REFERENCES}

1. Curtis S, Evens E, Sambisa W. Contraceptive discontinuation and unintended pregnancy: An imperfect relationship. Int Perspect Sex Reprod Health. 2011;37:58-66.

2. Parr NJ. Discontinuation of contraceptive use in Ghana. J Health Popul Nutr. 2003;21:150-157.

3. Trussell J. Contraceptive efficacy. In: Hatcher RA, T J, Nelson AL, Cates W, Stewart FH, Kowal D, eds. Contraceptive Technology. 19th edn. New York, NY: Ardent; 2007:759.

4. Ghana Statistical Service, Ghana Health Service, ICF Macro. Ghana Demographic and Health Survey 2014. 2015. https://dhsprogram.com/ pubs/pdf/fr307/fr307.pdf. Accessed February 12, 2017.

\title{
CONFLICTS OF INTEREST
}

The authors have no conflicts of interest.

\section{Single-incision laparoscopic abdominal cerclage placement: A retrospective study of single-port and robotic single-port versus multiport laparoscopy}

\author{
Yiming Zhang ${ }^{1,2}$ | Manisha Gandhi ${ }^{1}$ | Courtney Birchall ${ }^{1}$ | Xiaoming Guan ${ }^{1, *}$ \\ ${ }^{1}$ Department of Obstetrics and Gynecology, Baylor College of Medicine, Houston, TX, USA \\ ${ }^{2}$ Division of Reproductive Medicine, Jinan Central Hospital affiliated to Shandong University, Jinan City, Shandong Province, China \\ ${ }^{*}$ Correspondence \\ Xiaoming Guan, Department of Obstetrics and Gynecology, Baylor College of Medicine, Houston, TX, USA. \\ Email: xiaoming@bcm.edu
}

